\title{
Implementación de la estrategia didáctica del desarrollo del pensamiento crítico-reflexivo en el análisis literario de Hamlet de Shakespeare
}

\section{Implementation of the didactic strategy of critical-reflexive thinking in the literary analysis of Shakespeare's Hamlet}

\author{
Norman Marín-Calderón ${ }^{1}$ \\ Universidad de Costa Rica \\ Facultad de Letras \\ San José, Costa Rica \\ normanmarin@hotmail.com
}

Recibido: 4 febrero 2013 Aceptado: 26 junio 2014 Corregido: 30 junio 2014

\begin{abstract}
Resumen: Este ensayo se concentra en el proceso de ejecución del desarrollo del pensamiento críticoreflexivo como una estrategia didáctica universitaria en la enseñanza de una clase de literatura, más específicamente, de la obra de William Shakespeare, Hamlet (1603). Dicha estrategia se basó en una serie de actividades comunicativas que se propusieron observar el desarrollo de las habilidades cognitivas, el proceso de crítica y análisis, la reflexión personal a través de experiencias vivenciales, así como el desarrollo de técnicas de argumentación y pensamiento crítico de los estudiantes. El propósito en la implementación de la estrategia fue la de potenciar la calidad académica de las producciones críticas y argumentativas de los estudiantes, al momento de analizar Hamlet en tanto texto literario.
\end{abstract}

Palabras clave: Pensamiento, crítica, reflexión, vivencial, argumentación, Hamlet.

\begin{abstract}
This essay concentrates on the process of teaching execution in the critical-reflexive thinking development as a college didactic strategy when teaching literature, more specifically William Shakespeare's Hamlet (1603). Such a strategy is based on a series of communicative activities which analyze the development of students' cognitive abilities, their process of criticism and analysis, their personal reflection through experiential moments as well as the development of techniques on argumentation and critical thinking. The purpose of this strategic implementation was to improve students'academic quality in their critical and argumentative productions when analyzing Hamlet as a literary text.
\end{abstract}

Key Words: Thinking, criticism, reflection, experiential, argumentation, Hamlet.

El pensamiento crítico es una corriente reflexiva, razonable, que está centrada en decidir qué creer, qué hacer o qué pensar.

Robert H. Ennis

\footnotetext{
Psicoanalista, filólogo y educador. Doctor en Letras por Purdue University, Estados Unidos. Magíster en Psicoanálisis por el Instituto de Altos Estudios Universitarios de la Universidad de León, España y por el Centro de Investigaciones y Estudios Psicoanalíticos de la Fundación Mexicana de Psicoanálisis. Y Magíster en Literatura Inglesa por la Universidad de Costa Rica. Es profesor en la Facultad de Letras y el Sistema de Estudios de Posgrado de la Universidad de Costa Rica. Ejerce su práctica clínica psicoanalítica en San José de Costa Rica.
} 


\section{Introducción}

Este ensayo se elaboró a partir de una experiencia didáctica en el campo de los estudios literarios en el curso-seminario de licenciatura en Filología Española, FL-5129: Análisis de Hamlet y El rey Lear de Shakespeare de la Escuela de Filología, Lingüística y Literatura de la Facultad de Letras de la Universidad de Costa Rica. Es también el resultado de la implementación del desarrollo del pensamiento crítico-reflexivo, en tanto estrategia docentedidáctica, la cual se trabajó con la guía de la profesora Nora Cascante Flores en el curso FD-0340-02: Docencia Universitaria, durante el segundo semestre del año 2012.

El propósito de este ejercicio didáctico fue considerar un texto literario más allá de lo que las palabras mismas dicen; es decir, ahondar en el análisis crítico que puede resultar de una lectura introspectiva y metódica de este. Para ello, se idearon una serie de actividades comunicativas con el fin de abordar el drama de Hamlet a través del pensamiento críticoreflexivo, la aplicación de la experiencia vivencial, la técnica de la argumentación y la táctica del pensamiento socrático.

Entre las diferentes técnicas o estrategias docentes que existen para promover una lectura crítica de un texto literario, se optó por la del "desarrollo del pensamiento críticoreflexivo" porque se considera como aquella que más se acerca a una lectura extensiva y analítica, a saber, obra que va más allá de lo que el texto mismo articula.

Junto con la técnica de la argumentación, la estrategia del pensamiento crítico-reflexivo promueve la posibilidad de solucionar problemas de orden vivencial, los cuales activan en el cuerpo estudiantil el conocimiento aprendido previamente ahí donde también promueven la creatividad, incentivan el autoaprendizaje, estimulan el pensamiento crítico, así como la argumentación y la toma de decisiones; al mismo tiempo favorecen las habilidades de trabajo colaborativo e interpersonal. Por esta razón, y en consonancia con una didáctica comunicativa centrada en los y las estudiantes y, en el campo de los estudios literarios, es que la estrategia del desarrollo del pensamiento crítico-reflexivo se propone como la más idónea para promover la experiencia analítica de cada estudiante al leer e interpretar la obra dramática Hamlet de Shakespeare.

\section{El desarrollo del pensamiento crítico-reflexivo como estrategia didáctica universitaria: Bases teóricas}

\section{Apertura}

El desarrollo del pensamiento crítico-reflexivo es una técnica didáctica-docente centrada en el/la estudiante y su propio modo de aprendizaje. Se puso en práctica con el fin de preparar al alumnado con el propósito de que pudiera apre(he)nder por sí mismo y ser capaz de dirigir su propio modo de aprendizaje. Es una técnica basada en el descubrimiento, el análisis y la participación de los mismos estudiantes, la cual establece las condiciones para que, a través del conocimiento aprendido anteriormente, desarrollen habilidades analíticas innovadoras, organicen juicios de valor crítico y alcancen estrategias para aplicar lo aprendido en su vida 
cotidiana. Esta estrategia les sirvió a los estudiantes para resolver problemas de carácter crítico y vivencial. Del mismo modo, el empleo de esta estrategia propició en cada estudiante un aprendizaje crítico, reflexivo e intencional. El objetivo final en la aplicación de dicha técnica se construyó ante la premisa de que esta estrategia didáctica le ofrece al estudiante cierta "autonomía" en la búsqueda de su propio saber así como el conocimiento académico en general.

\section{Estado de la cuestión}

Existen diferentes estrategias, tácticas y técnicas didácticas construidas con el fin de activar y mejorar las distintas habilidades de pensamiento en el estudiantado (universitario). Según Muria y Damián (2003), existen tres tipos de habilidades del pensamiento que resultan en los metacomponentes de cualquier tipo de ejecución cognitiva, a saber: "(1) Las habilidades de pensamiento crítico-analítico, [las que] incluyen actividades tales como analizar, criticar, juzgar, evaluar y contrastar. (2) Habilidades de pensamiento creativo, [que] incluyen crear, descubrir, inventar, imaginar, suponer e hipotetizar. Y finalmente (3) las habilidades de pensamiento práctico, [que] incluyen aplicar, usar, utilizar y practicar" (p. 161). Una atinada combinación de estos tres tipos de habilidades del pensamiento es la que se explora en este proyecto con el fin de aplicar la estrategia del desarrollo del pensamiento crítico-reflexivo en el análisis literario del drama Hamlet de Shakespeare.

Por consiguiente, hay que considerar todas aquellas variables que intervienen en la enseñanza del material literario por medio de las diferentes habilidades del pensamiento consideradas anteriormente. Según Claxton (1999), para que la enseñanza de estas habilidades de pensamiento — crítico-analítico, creativo y práctico — tomen lugar, habría que considerar la necesidad de enseñar de manera paralela a la estrategia, todo aquel material relacionado con la instrucción sobre qué es pensar así como las habilidades de aprendizaje para adquirir dicho conocimiento. De la misma manera, se debe instruir al estudiante sobre todas esas habilidades metacognitivas que contribuyen a la transmisión de las habilidades de este tipo de pensamiento a otras situaciones vivenciales y contextos intersubjetivos, relacionados con los aprendientes.

Con el fin de poder implementar dicha estrategia en la enseñanza de las habilidades de pensamiento, Muria y Damián (2003) proponen nuevamente tomar en cuenta los siguientes elementos didácticos: (1) Las modalidades de enseñanza que se remiten a las actividades pedagógicas empleadas, tales como los seminarios teóricos y de investigación, talleres presenciales, discusión de grupo y debates así como el trabajo cooperativo, entre otros. (2) Las estrategias de enseñanza que promueven una manera más personalizada de enseñanza allí donde el docente se torna en un modelo de aprendizaje. (3) Las estrategias de aprendizaje deben guiar al estudiante a que escoja y aplique aquella información que mejor se ajuste a su estilo de aprendizaje. Las autoras mencionan técnicas como el análisis, la clasificación, la aplicación, la síntesis, la elaboración así como una "evaluación crítica y reflexiva en su aprendizaje [del estudiante] en general y en la solución de problemas de manera creativa" (p. 162). Finalmente, también influye (4) el clima psicológico en donde siempre se promuevan ambientes democráticos, cooperativos, "fomentando en todo momento la autonomía de los 
estudiantes y el respeto a los derechos humanos" (p. 162). Es decir, mientras el/la estudiante aprende la materia del curso, también está ensayando cómo pensar, de qué manera analizar y cómo evaluar el material cognitivo propuesto.

\section{El pensador crítico ideal}

El propósito esencial en el manejo de esta estrategia didáctica es la de leer y analizar un texto literario mediante un modelo de pensamiento que promueva el análisis, la crítica y la reflexión. De hecho, se estima que esta estrategia puede mejorar las condiciones de lectura crítica de los estudiantes de literatura desde un contexto de reflexión y experiencias vivenciales. El deseo último de este proyecto radica en formar, no tanto a un lector implicado, sino más bien, a un "pensador crítico ideal":

El pensador crítico ideal es una persona habitualmente inquisitiva; bien informada, que confía en la razón; de mente abierta; flexible; justa cuando se trata de evaluar; ... prudente al emitir juicios; ...clara respecto a los problemas o las situaciones que requieren la emisión de un juicio; ordenada cuando se enfrenta a situaciones complejas; diligente en la búsqueda de información relevante; razonable en la selección de criterios; enfocada en preguntar, indagar, investigar; persistente en la búsqueda de resultados tan precisos como las circunstancias y el problema o la situación lo permitan. (Facione, 2007, p. 21)

En suma, lo más significativo en la formación de un "pensador crítico ideal" es enseñarle a apropiarse de los conceptos y principios básicos de un determinado tema o área de conocimiento, para luego llegar a dominar dicha materia de manera crítica, reflexiva, analítica y vivencial ahí donde sujeto y entorno se hacen uno.

\section{Mayéutica: El modelo socrático del pensamiento}

La estrategia del desarrollo del pensamiento crítico-reflexivo se basa en una "personalización" del proceso enseñanza-aprendizaje que se caracteriza por su perfil activo de aprendizaje, la interrelación entre la experiencia subjetiva de este y la colectiva, así como la exigencia de activar el autoaprendizaje. Para Montes de Oca y Machado (2011), la intención del empleo de esta estrategia radica en el beneficio que puedan proveer los métodos seleccionados, los cuales deberían estar "orientados a propiciar el cuestionamiento, la actitud de búsqueda, el procesamiento de la información, el reconocimiento de la propia identidad, el aprender a autoregularse, el desarrollo de un pensamiento crítico y la solución de problemas" (p. 483). De aquí que esta estrategia destacada resalte entre las otras existentes debido a la posibilidad que brinda en el campo de la socialización y el enriquecimiento del conocimiento académico (literario); y que, a su vez, surge como el producto de relaciones críticas intersubjetivas en contextos académicos e igualmente vivenciales. Con el fin de promover este tipo de habilidades, la mayéutica —antiguo método socrático- sería uno de los más idóneos para suscitar el pensamiento crítico-reflexivo, corriente caracterizada por los ejercicios de creatividad y análisis "profundo".

La enseñanza socrática — la mayéutica — es una de las técnicas didácticas más antiguas 
en la historia de la humanidad. Se basa en el cuestionamiento personal como modo para acceder a la "verdad" del conocimiento. Promueve el pensamiento crítico por medio de la reflexión mediatiza entre el sujeto que se pregunta y el entorno que inquiere. El fin último de esta técnica es la de moldear una mente inquisitiva, crítica y analítica por medio de preguntas abiertas y formales.

Escarbar, preguntar, explorar, considerar, cuestionar, analizar, inquirir, criticar, involucrar, articular, entre otros, son verbos que se toman en cuenta en el momento de la aplicación de esta técnica pedagógica antigua. La base de la enseñanza socrática consiste en preguntarle e insistirle al sujeto con el fin de que él o ella misma descubra las respuestas de sus propias interrogantes, siempre mediante el cuestionamiento constante. De acuerdo con lo elaborado por Paul y Elder (1997), existen seis tipos de preguntas que promueven el aprendizaje crítico a través de contextos indagadores constructivos, a saber: (1) preguntas conceptuales aclaratorias [¿Puede darnos un ejemplo de lo que usted dice?], (2) interrogaciones para comprobar conjeturas [Por favor, explique por qué dice eso], (3) preguntas que exploran evidencias [¿Qué evidencia existe para apoyar lo que usted está diciendo?], (4) interpelaciones ante otras perspectivas y puntos de vista [¿De qué otras maneras alternativas se puede mirar esto?], (5) preguntas para comprobar implicaciones y consecuencias [¿Cuáles son las consecuencias de esas conjeturas?], e (6) inquisiciones sobre otras preguntas [¿Por qué cree usted que formulé esa pregunta?].

En fin, en el método socrático de enseñanza lo que se busca es escrutar diferentes niveles y dimensiones del pensamiento de los estudiantes, por medio de ejemplos y vivencias concretas, siempre indagando mediante preguntas investigativas significativas. La premisa básica de este tipo de enseñanza radica en la posibilidad de que es el mismo alumno quien debe llegar al dominio de su pensamiento crítico mediante la reflexión profunda y analítica de un tema. Para lograrlo, el docente, quien es un simple facilitador del proceso enseñanza-aprendizaje, debe promover la discusión, el análisis, el debate fructífero, la escritura y las conexiones que potencialmente existan entre el material estudiado y las experiencias vivenciales de cada uno de los sujetos circunscritos en dicho proceso.

\section{El arte de la argumentación}

El arte de la argumentación conlleva un manejo prolijo y sustancial de la lengua. De hecho, el lenguaje se convierte en el instrumento simbólico por excelencia que utilizan estudiantes y docentes para interactuar y así acceder a cierto material significativo, para luego compartirlo y negociarlo con su entorno humano, subjetivo y social (De Longhi, 2000). Según Cuenca (1995), la argumentación se define como una forma de interacción comunicativa $\mathrm{y}$ vivencial entre diferentes y variados sujetos quienes confrontan sus saberes, sus puntos de vista y experiencias sobre un tema particular, con el propósito mismo de convencer al otro mediante un lenguaje insertado dentro de un código comunicativo en consenso. Al respecto, Campaner y De Longhi (2007) afirman:

El proceso de argumentación, contextualizado en un modelo de problematización guiada, promueve, orienta y permite ir enriqueciendo el diálogo entre los diferentes actores de la clase desde el debate o discusión. Dicho discurso, en el marco de una estrategia de juicio, es 
fundamentalmente argumentativo, de formulación, análisis y confrontación de textos orales y escritos. Allí los participantes, asumiendo un rol definido, se comprometen con una posición, afrontan la problemática y desde allí defienden estratégicamente su postura. A esta particular forma de enseñar [ciencias] le llamamos Estrategia Didáctica Argumentativa (EDA) y requiere dos condiciones básicas: un abordaje interdisciplinario (desde la lengua y desde la ciencia) y tomar el contenido procedimental de argumentación como contenido a enseñar. (p. 446)

Lo que confirma esta aserción es que, al enseñar literatura, también debe entrenarse al estudiantado para aprender a pensar y hablar, de acuerdo con los postulados definitorios de la argumentación, el pensamiento crítico y la reflexión vivencial. Por lo tanto, es imperativo enseñar el procedimiento para adquirir el hábito y desarrollo del pensamiento crítico-reflexivo y la argumentación mientras simultáneamente se enseña el contenido de los estudios literarios, con rúbricas específicas de crítica, análisis, discusión e intervenciones textuales estratégicas.

\section{Cierre}

El propósito en la implementación de la estrategia del desarrollo del pensamiento críticoreflexivo por medio de técnicas didácticas tales como la mayéutica, la argumentación y su relación con elementos y experiencias vivenciales les facultó a los estudiantes tomar una postura personal y vivencial que defender, durante la lectura y el análisis literario de Hamlet de Shakespeare. Esto les posibilitó defender una posición crítica y ética, debatir problemas actuales, negociar posibles soluciones y deliberar sobre la relación viable que existe entre la ficción del texto y la realidad de su vida cotidiana.

\section{¿Cómo enseñar literatura utilizando la estrategia del pensamiento crítico-reflexivo?: Experiencia, implementación y valoración de los aprendizajes}

Esta experiencia pedagógica de lectura crítica y análisis reflexivo pretendió que el estudiantado fuera capaz de analizar, desde una perspectiva cavilosa e introspectiva, los elementos literarios y metacognitivos del drama Hamlet de William Shakespeare. Para esto (1) se analizó Hamlet por medio de discusiones y debates que estimularon la producción intelectual crítica; (2) se criticaron ciertos modelos vivenciales presentes en la obra dramática por medio de la argumentación y la toma de posiciones; (3) se flexionaron los elementos extraliterarios presentes en Hamlet por medio del ejercicio del método socrático; y también (4) se profundizaron las posibilidades vivenciales que existen entre el texto literario (ficcional) y la realidad contemporánea (real).

\section{Etapas en la planificación de las actividades docentes}

La táctica empleada para poner en práctica esta estrategia docente se diseñó con el fin de promover un "aprendizaje estratégico" de modo tal que los estudiantes elijan, planifiquen, coordinen y apliquen los modelos aprendidos durante el curso, además de todos aquellos procedimientos necesarios para analizar literariamente el drama de Hamlet.

Por su parte, las tácticas utilizadas en clase consistieron principalmente en preguntas 
guiadas de análisis, esquemas de estructuración de textos, discusión de grupo, métodos de situación y otras técnicas participativas, tales como el debate y el aprendizaje colaborativo.

Así, las diferentes tácticas de pensamiento crítico-reflexivo utilizadas en este curso incluyen, entre otras:

(a) Considerar la propia vida (realidad) como punto de partida;

(b) Posibilitar la resignificación del sujeto con su entorno;

(c) Insistir en el análisis del texto literario más allá de lo que dice explícitamente;

(d) Concienciar al alumnado a que "profundice" y ponga su atención en aquello del texto que no se dice o que se dice "a medias".

De igual manera, la implementación de la estrategia del desarrollo del pensamiento crítico-reflexivo favoreció al estudiante a explorar la posibilidad de ver en el texto literario un campo para el análisis y solución de problemas vivenciales reales. Este punto de experiencia subjetivo se llevó a cabo mediante esta secuencia de eventos formulada dentro de la clase misma. Viñetas de experiencia:

- Detección de un problema a resolver y aproximación a dicho problema por medio de la valoración teórica;

- Localización del origen del problema; señalamiento de sus posibles soluciones o al menos sus más rigurosos acercamientos;

- Realización de un debate (aprendizaje colaborativo e interpersonal); promoción de la confrontación responsable y constructiva entre los diferentes puntos de vista de la clase;

- Finalmente, la elaboración y análisis de las conclusiones del caso y la valoración de lo aprendido a lo largo de la lectura de Hamlet de William Shakespeare.

Finalmente, dicha planificación incluyó aspectos de relevancia tanto académica como vivencial, a saber, la promoción de un proceso de aprendizaje basado en el diálogo reflexivo, primero y participativo después, la valoración de la posibilidad de realizar una interpretación crítica-literaria a partir de expresiones no verbales, y finalmente, la promoción de la escritura como modo adecuado de reflexión, cotejo y auto-aprendizaje.

\section{Metodología}

1. En primer término, los estudiantes leen Hamlet, luego:

(a) Los alumnos lo comentan en grupo, de forma oral, acto por acto;

(b) Inmediatamente después, el docente introduce los temas principales del drama por medio de una clase magistral que apoya con un PowerPoint y algunos bosquejos/resúmenes de acompañamiento (Temas: la venganza, el parricidio, el incesto, el amor, la muerte, la locura, el complejo freudiano del Edipo, el acto, la duda y la certidumbre, entre otros); 
(c) Posteriormente, los y las estudiantes responden oralmente, en grupos pequeños (no más de cuatro personas por grupo) las "preguntas guías" elaboradas por el profesor (véase las preguntas guías que incluyen cuestiones relacionadas con los personajes, la trama y algunos temas de carácter universal, contenidas en el Apéndice \# 1 de este ensayo).

2. En previas clases, el docente ha presentado dos textos críticos sobre Hamlet: (a) "Hamlet, un caso clínico" (1979) de Jacques Lacan y (b) Hamlet y sus críticos (1969) de Margarita Quijano. Los estudiantes leyeron y estudiaron este material con suficiente anterioridad para poder implementar la estrategia durante las horas de clase.

3. En un último momento se realizó un análisis de los dos textos críticos por medio de:

(a) Una exposición docente (un análisis guiado por parte del docente);

(b) Diferentes preguntas directas a los estudiantes mediante el "método socrático";

(c) La realización de actividades de discusión en grupos pequeños por medio de la argumentación y el debate constructivo; y

(d) La comparación entre la obra ficcional de Shakespeare y la vida cotidiana de los estudiantes (lo vivencial). En esta etapa, la guía de análisis y reflexión que el docente introdujo fue de suma importancia en la compresión del material de crítica.

\section{Elementos vivenciales del proyecto}

En el drama shakespeariano, al que se ajusta este proyecto de investigación, se pueden identificar los siguientes elementos que requieren un acercamiento analítico-vivencial: el problema de la locura, el incesto, la verdadera amistad, la enfermedad y la podredumbre, el amor (imposible), la muerte, las relaciones filiales, el efecto cómico de la tragedia y la realidad psíquica, entre otros.

\section{Evaluación y valoración de los aprendizajes}

La evaluación y valoración final de la estrategia se realizó mediante un ensayo de tres a cinco páginas en donde el estudiante escogió uno de los "elementos vivenciales" relacionándolo tanto con el material crítico de Lacan o Quijano, y con la vida cotidiana de cada uno de ellos. Fue una composición escrita durante dos horas de clase. Al final de las dos horas, los estudiantes compartieron las ideas desarrolladas en su ensayo con el resto de la clase, en forma oral. Luego el instructor valoró el trabajo de acuerdo al contenido expuesto, para después devolvérselos con comentarios y observaciones críticas pertinentes.

\section{Conclusiones y resultados de la experiencia educativa}

La implementación de esta estrategia didáctica sirvió para lograr que los estudiantes de literatura encontraran maneras críticas y analíticas de leer/estudiar Hamlet, por medio de técnicas significativas de análisis literario, tales como el pensamiento crítico, la técnica del análisis socrático, el arte de la argumentación y los principios del "pensador crítico ideal".

El resultado de este proyecto se puede resumir con la reflexión de Stephen Toulmin 
quien asevera que "el objetivo de estos estudios es traer problemas, no solucionarlos; para llamar la atención hacia un campo de cuestionamientos, más que solo contemplarlo; y para provocar la discusión, más que servir como un tratado sistemático" (nuestra traducción, p. 41).

El mayor de los logros obtenidos en esta serie de clases, por medio de la estrategia del desarrollo crítico-reflexivo, fue la capacidad de desarrollar modelos de entendimiento y razonamiento lógico-constructivos que amplificaron los estudiantes en el análisis literario de Hamlet. Se logró "convencer" al estudiante que a Shakespeare se le puede leer desde parámetros que no son exclusivamente literarios, sino que se ubica como un drama universal, precisamente porque se puede vislumbrar desde otros puntos de vista, en este caso, desde temas como la locura, el deseo inconsciente y el amor imposible, entre otros.

Finalmente, el uso de esta estrategia ayudó a que los estudiantes no se "dispersaran" mientras leían, promoviendo mucha creatividad al desarrollar su pensamiento crítico así como su elaboración reflexiva que le ofreció una significativa explicación y análisis del texto literario.

La (auto) evaluación de la estrategia (Apéndice 2), por parte de los mismos estudiantes, avala la valiosa ejecución de la estrategia como técnica necesaria para leer y comprender un texto literario. Así por ejemplo, 89\% por ciento de los estudiantes respondieron el ítem uno de la encuesta en las categorías de seis y siente, siendo siete (7) la más alta de las calificaciones. La más baja de las rúbricas se ve representada en la pregunta cinco en donde solo un 71\% están satisfechos con "los niveles de asimilación y compromiso personal y vivencial con los temas abordados". Por su parte, la más alta de las calificaciones de autoevaluación se encuentra ubicada en la pregunta tres y nueve: primero, la pregunta tres — "grado de motivación del facilitador" —alcanza un $94 \%$ de aceptación entre las notas de seis y siete. Lo mismo ocurrió con la pregunta nueve — cuestión relacionada con la experiencia de que si "esta estrategia es útil para la consecución de otros cursos de mi carrera". El 91\% de los estudiantes encuestados valoraron este ítem entre los seis y siete puntos. Este escrutinio de autoevaluación nos permite vislumbrar el grado de aprovechamiento que la mayoría de los estudiantes pudo obtener, al analizar Hamlet, desde una perspectiva comunicativa de desarrollo del pensamiento crítico-reflexivo.

Después de todo, este proceso permitió que el estudiantado leyera la obra dramática, pero "entre líneas", es decir, yendo un poco más allá de lo que las meras palabras plasmadas en el texto de Shakespeare decían. La posibilidad de crítica, análisis y reflexión hicieron que la obra shakespeariana se convirtiera en un compendio de experiencias reales y significativas para cada aprendiente. Y en eso radica el arte de convertir las meras palabras que se articulan en un acto fundacional. Bien lo apunta el maestro renacentista:

- Polonio: “¿Qué estáis leyendo, mi señor?”

- Hamlet: "Palabras, palabras, palabras." 


\section{Apéndice 1}

\section{PREGUNTAS GUIADAS DE ARGUMENTACIÓN Y CRÍTICA}

\section{Seminario:}

FL-5129: Análisis de Hamlet y El rey Lear de Shakespeare

Facilitador: Norman Marín Calderón

-Fecha: 22/11/12

En grupo de cuatro personas, analice las siguientes situaciones siguiendo como modelo la estrategia de aprendizaje "Desarrollo del pensamiento crítico-reflexivo", tal y como lo estudiamos en clase. Emplee los textos teóricos de Lacan y Quijano como base para su análisis crítico.

(1) Desarrolle y comente estas preguntas o temas:

(a) Rosencrantz y Guilderstern han sido descritos como "medios-hombres". ¿En qué medida puede justificar usted esa aserción?

(b) ¿Hasta qué punto y en qué sentido es el diálogo (cómico) entre los dos sepultureros (Acto V, Escena 1, pág. 179 en adelante), funcional y necesario en el desarrollo dramático de esta precisa acción (seria)?

(2) Desarrolle los siguientes temas basados en la táctica de la reflexión y sus elementos vivenciales:

(a) La enfermedad y la podredumbre

(b) El amor y la muerte

(3) Responda la siguiente pregunta compuesta siguiendo los parámetros del arte de la argumentación:

(a) ¿Qué relación existe entre estas tres escenas y el tema o la acción principal del drama Hamlet?

(b) La alabanza sincera de Hamlet a Horacio (acto III, escena 2, pág. 109)

(c) La intervención de Claudio: “iQué duro latigazo dan a mi conciencia estas palabras!” (acto III, escena 1, pág. 99 en adelante).

(d) Las citas textuales de Eneas a Dido recitadas por los actores transeúntes (acto II, escena 2, pág. 89 en adelante).

(4) Escriba un ensayo bien redactado, entre las cuatro y cinco páginas, en donde usted proponga una solución práctica y bien fundamentada para uno de los temas que se le presentan en esta guía, en la pregunta \# (2). 


\section{Apéndice 2}

\section{AUTOEVALUACIÓN DE LA ESTRATEGIA}

\section{Seminario:}

FL-5129: Análisis de Hamlet y El rey Lear de Shakespeare

Facilitador: Norman Marín Calderón

Fecha: 22/11/12

Por favor, conteste de la manera más honesta las siguientes proposiciones. No es necesario que escriba su nombre. Toda sugerencia adicional que nos aporte se la agradeceremos con el fin de realizar los mejoramientos pertinentes en las próximas implementaciones. Por favor, evalúe en la escala 1-7, siendo 1 la menor calificación y 7 la mayor.

¡Muchas gracias!

UTILIDAD DE LOS CONTENIDOS ABORDADOS EN LA ESTRATEGIA. Importancia y utilidad que han tenido para usted los temas tratados en el curso utilizando la técnica del "pensamiento crítico-reflexivo":

$$
\begin{array}{llllllll}
1 & 2 & 3 & 4 & 5 & 6 & 7
\end{array}
$$

METODOLOGIA UTILIZADA EN LA ESTRATEGIA. Respecto a los métodos y estrategias utilizadas por el facilitador para impartir los contenidos de análisis crítico de Hamlet fue:
1
2
3
4
5
6
7

GRADO DE MOTIVACIÓN DEL FACILITADOR. Nivel de participación y de motivación ofrecido por el facilitador por medio de experiencias vivenciales fue:

$\begin{array}{llllllll}1 & 2 & 3 & 4 & 5 & 6 & 7\end{array}$

CLARIDAD DE LA EXPOSICIÓN. Respecto al lenguaje y orden dado a la estrategia y su utilidad en el desarrollo del pensamiento analítico:

$\begin{array}{llllllll}1 & 2 & 3 & 4 & 5 & 6 & 7\end{array}$

NIVEL DE ASIMILACION Y COMPROMISO PERSONAL Y VIVENCIAL CON LOS TEMAS ABORDADOS.

Evalúese a usted mismo en el grado de motivación e interés personal para atender y seguir la clase y sus actividades cuando se implementó la táctica del "pensamiento crítico-reflexivo" para analizar Hamlet:
1
2
3
4
5
6
7

CALIDAD DEL MATERIAL LITERARIO Y TEÓRICO ENTREGADO PARA ABORDAR LA ESTRATEGIA:
12
$3 \quad 4$
$5 \quad 6$
7

INFRAESTRUCTURA Y COMODIDAD DEL LUGAR PARA REALIZAR LAS ACTIVIDADES COMUNICATIVAS DE ARGUMENTACIÓN Y CRÍTICA:

$\begin{array}{llllllll}1 & 2 & 3 & 4 & 5 & 6 & 7\end{array}$




\section{CALIDAD Y CLARIDAD DE LOS EJEMPLOS ENTREGADOS PARALA CRÍTICA Y REFLEXIÓN DE HAMLET:

$\begin{array}{lllllll}1 & 2 & 3 & 4 & 5 & 6 & 7\end{array}$

LA EXPERIENCIA DE ESTA ESTRATEGIA ES ÚTIL PARA LA CONSECUSIÓN DE OTROS CURSOS DE MI CARRERA:

$\begin{array}{llllllll}1 & 2 & 3 & 4 & 5 & 6 & 7\end{array}$

\section{SUGERENCIAS Y COMENTARIOS PARA EL MEJORAMIENTO DE LAS PRÓXIMAS ACTIVIDADES DE IMPLEMENTACIÓN EN EL DESARROLLO DEL PENSAMIENTO CRÍTICO-REFLEXIVO:}

\section{Referencias}

Claxton, G. (1999). Aprender. El reto del aprendizaje humano. Barcelona: Paidós.

Cuenca, M.J.(1995). Mecanismos lingüísticos y discursivos de la argumentación. Comunicación, Lenguaje y Educación, 25, 23-40. DOI: http://dx.doi.org/10.1174/021470395321340411

De Longhi, A.L., Ferreyra, A., Paz, A., Bermúdez, G., Solís, M., Vaudagna, E. y Cortés, M. (2005). Estrategias didácticas innovadoras para la enseñanza de las ciencias naturales en la escuela. Argentina: Universitas.

Facione, P.A. (2007). Pensamiento crítico: ¿Qué es y por qué es importante? Nueva York: Insight Assessment.

Montes de Oca, N. y Machado Ramírez, E. (2011). Estrategias docentes y métodos de enseñanzaaprendizaje en la educación superior. Humanidades Médicas 11(3), 475-488. Recuperado de http://www.humanidadesmedicas.sld.cu/index.php/hm/article/view/127/68

Muria Vila, I. y Damián Díaz, M. (2003). La enseñanza de las habilidades de pensamiento desde una perspectiva constructivista. Umbral. Revista de Educación, Cultura y Sociedad III(4), 158-163.

Lacan, J. (1983). Hamlet: un caso clínico. En Lacan oral. Buenos Aires: Editorial Xavier Bóveda.

Quijano, M. (1962). Hamlet y sus críticos. México: Editorial UNAM.

Paul, R. y Elder, L. (1997). Critical Thinking [Pensamiento crítico]. California: Foundation for Critical Thinking.

Shakespeare, W. (2009). Hamlet, Príncipe de Dinamarca. (1603). Madrid: Cátedra. DOI: http://dx.doi.org/10.1017/CBO9780511704130

Toulmin, S. (2003). The Uses of Arguments. Nueva York: Cambridge University Press. DOI: http://dx.doi.org/10.1017/CBO9780511840005 